\title{
Analysis of Long-living Plasmoids at Atmospheric Pressure
}

\author{
Stefan Noack ${ }^{*, \dagger}$, Alex Versteegh ${ }^{*, * *}$, Burkhard Jüttner* and Gerd Fussmann ${ }^{*, \hbar}$ \\ *AG Plasmaphysik, Humboldt-Universität zu Berlin, Newtonstr. 15, 12489 Berlin, Germany \\ ${ }^{\dagger}$ Fakultät für Physik und Geowissenschaften, Universität Leipzig, Linnéstr. 5, 04103 Leipzig, Germany \\ ** Eindhoven University of Technology, P.O. Box 513, 5600 MB Eindhoven, The Netherlands \\ ${ }^{\ddagger}$ Max-Planck-Institut für Plasmaphysik, EURATOM Association, Germany
}

\begin{abstract}
Ball-like plasmoids were generated by discharging a capacitor bank via a water surface. In the autonomous stage after current zero they have diameters up to $0.2 \mathrm{~m}$ and lifetimes of some hundreds milliseconds. They were studied by applying high speed cameras, spectroscopy and an array of lasers. The latter allows to determine the index of refraction and thus give information on the internal structure of the plasmoids.
\end{abstract}

\section{INTRODUCTION}

Some years ago G.D. Shabanov and colleges reported on the generation of luminous plasmoids at atmospheric pressure produced from discharges along water surfaces [1,2]. The objects attracted particular interest because of their possible relation to ball lightning. More recently the same authors described the objects as consisting of rather cold hydrated water clouds having temperatures around $330 \mathrm{~K}$ [1] that unlikely contained any noticeable concentration of free electrons [2]. Our group in Berlin succeeded in generating plasmoids using the same mechanism of production, but came to quite a different assessment with respect to the consistence of the luminous balls. The plasmoids are found to consist of a true plasma confined by a cold envelope. Analyzing the Stark-broadening of various lines from atomic species the electron densities were found to be in the range of $10^{20} \ldots 10^{22} \mathrm{~m}^{-3}$. Similarly, the electron temperature is estimated to be $0.2 \ldots 0.4 \mathrm{eV}$ from line ratio intensities and molecular rotation excitation. The energy sources for the luminescence seem to be provided by chemical reactions. Most of our findings have already been reported in two recently published papers $[3,4]$. In the present work we concentrate on some particular investigations that, in the first place, corroborate our previous statements about temperatures of the electrons and the neutrals and may clarify some of the still open questions.

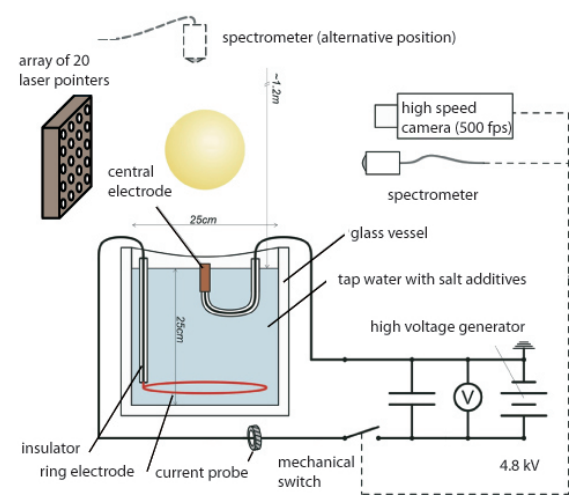

FIGURE 1. Experimental Setup

\section{EXPERIMENTAL SETUP}

Figure 1 shows the scheme of the experimental arrangement. It is similar to that in the references as far as the discharge system is concerned. The cylindrical discharge vessel made of glass is filled with tap water with optional additives of 
salts $\left(\mathrm{NaCl}, \mathrm{CaCl}_{2}\right)$. A copper ring at the bottom of the vessel serves as anode, while the grounded cathode is the isolated central electrode protruding some millimetres above the water surface. A capacitor bank of $1 \mathrm{mF}$ charged to $4.8 \mathrm{kV}$ is switched to the anode initiating a discharge with currents of order 30 to $100 \mathrm{~A}$. After $100 \ldots 150 \mathrm{~ms}$ the current is quenched to obtain an autonomous object without any external energy supply. In addition to the diagnostics shown in Fig. 1 thin thermocouples are used to measure the gas temperature. The latest achievement is an array of 4x5 diode lasers $(\lambda=650 \mathrm{~nm})$ allowing to perform space resolved measurements of the index of refraction by recording the deflection of the beams. In what follows we report on first measurements of this type where only a reduced number of the beams were taken into account.

\section{RESULTS}

\subsection{Spectroscopy}

Figure 2 a shows typical spectra taken with the high resolution Echelle spectrometer. As to be seen from the figure, the spectrum is dominated by lines from $\mathrm{K}, \mathrm{Na}, \mathrm{Ca}, \mathrm{Mg}$ (constituents of water) and $\mathrm{Cu}$ (central electrode) during the initial phase $(\mathrm{t}=15 \mathrm{~ms})$. Note that in case of $\mathrm{Ca}$ and $\mathrm{Mg}$ also the single ionized species CaII and MgII do occur. Later in the discharge $\mathrm{OH}$ and $\mathrm{CaOH}$ molecules also contribute considerably to light emission. Actually, they become dominant towards the end of the luminous phase at about $t=0.3 \mathrm{~s}$. Special interest has been devoted to the CaI emission lines that allow a determination of the electron temperature from a Boltzmann plot. Temperatures obtained this way (using transitions of type ${ }^{3} \mathrm{P}^{o}-{ }^{3} \mathrm{~S}$ and ${ }^{3} \mathrm{P}^{o}-{ }^{3} \mathrm{P}$ ) are plotted in Fig. $2 \mathrm{~b}$ as a function of time. They are seen to decay from initially $T_{e}=0.4 \mathrm{eV}=4640 \mathrm{~K}$ to about half that values after $90 \mathrm{~ms}$.

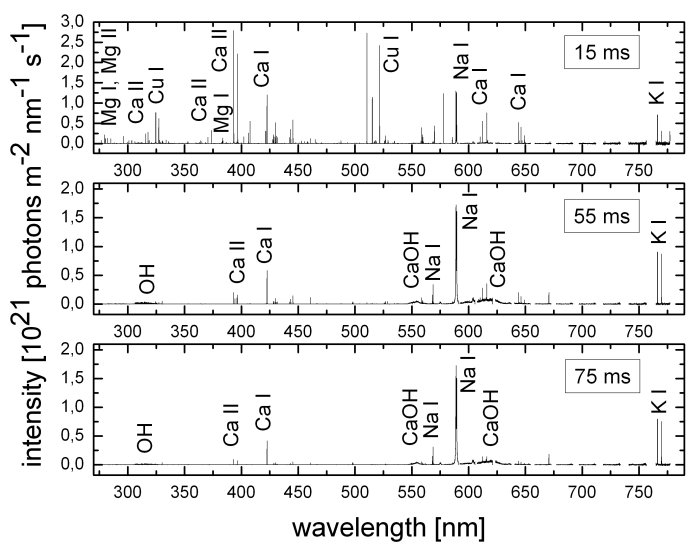

FIGURE 2. a) Time developed spectra b) Time dependence of electron temperature

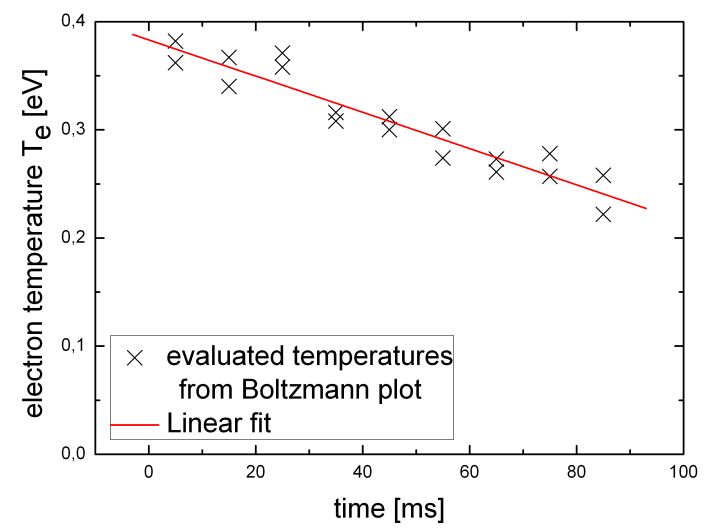

\subsection{Laser deflection measurements}

The laser beams are deflected when the plasmoid crosses their paths. The effect is easily detectable: we observe shifts of order $1 \mathrm{~cm}$ of the rest points on a plane wall in $5 \mathrm{~m}$ distance, i.e. deflection angles of order $\delta=2 \mathrm{mrad}$. The motion of the points is pursued by means of a high speed camera that records an image of all 20 beams every 2 ms. An example for a selection of four points at three different times is shown in Fig. 3. The different beams are seen to be deflected markedly at different times. The general behavior is a sudden large shift followed by a slow motion back to the rest position (black dots). Then a second motion (turbulent) is seen and and the return to the rest position. The large initial shift coincides with the time when the particular beam gets in contact with the outer boundary of the ball. It is thus also possible to determine the rising velocity of the ball by identifying the corresponding times for the five beams arranged in a distance of $3 \mathrm{~cm}$ along the central vertical axis. From the measured time intervals of $30 \mathrm{~ms}$ a rising velocity of $1 \mathrm{~m} / \mathrm{s}$ is deduced. This velocity is close to value stated previously [3], deduced from an image analysis of 
the balls. The deflection appears to be rather insensitive with regard to wavelength. This was checked by combining the rays of a red and a green laser by means of a beam splitter. The points of both beams stayed in coincidence at all times during the discharge.

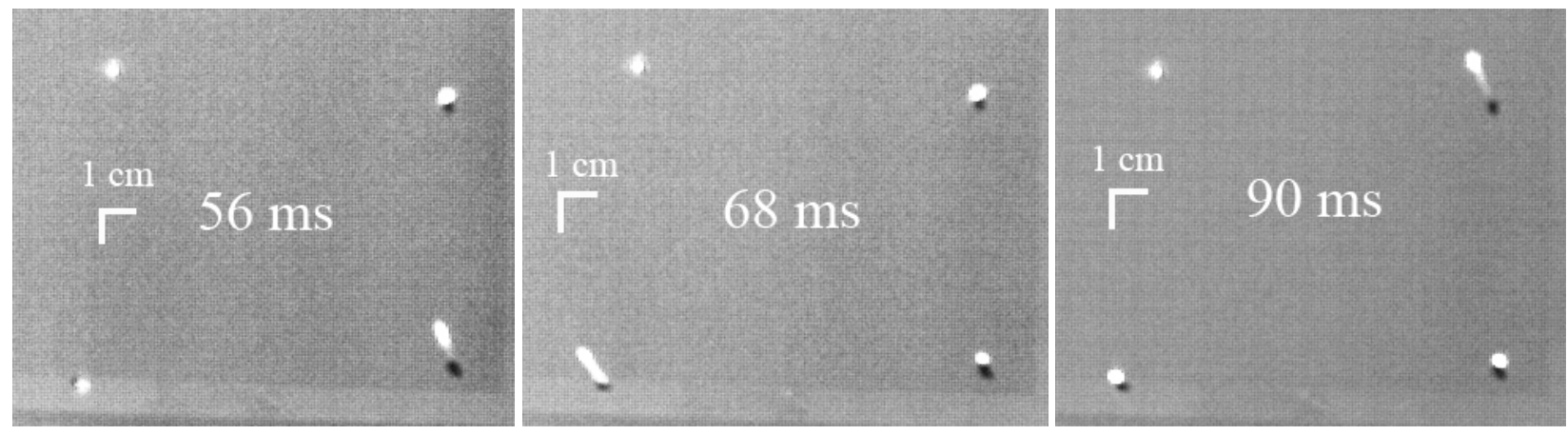

FIGURE 3. Deflection of the laser beams (section of the array)

Quantitative analysis of the beam deviation is provided by solving the equation for the light path $d / d s(N \vec{n})=\nabla \mathrm{N}$ where $\mathrm{N}$ is the index of refraction, $\vec{n}$ is a unit vector in the beam direction and $s$ is the length of arc [5]. Considering the plasma ball as a spherically symmetric object of radius $r=a$ and $N=N(r)$ the following equation

$$
\delta(\rho)=2 \rho \int_{\rho}^{a} \frac{d N}{d r} \frac{d r}{\sqrt{r^{2}-a^{2}}}
$$

can be derived for small deflection angels $\delta$. Here $\rho$ is the "collision parameter" (Fig. 4 b). From the general theory of Abel inversion the radial profile of the index of refraction is found to be

$$
N(r)=N_{a i r}-\frac{1}{\pi} \int_{r}^{a} \frac{\delta(\rho)}{\sqrt{\rho^{2}-r^{2}}} d \rho .
$$

The detected angles $\delta$ are found to be all positive, i.e. the plasmoid behaves like a dispersing lens. Consequently, $N_{\text {air }}>N_{\text {ball }}$ is to be postulated. In general, the refraction index of gases is frequency dependent and the following relation is to be found in the textbooks

$$
N^{2}(\omega)=1+\sum_{\alpha} \frac{n_{\alpha} e_{\alpha}^{2}}{\varepsilon_{0} m_{\alpha}\left(\omega_{\alpha}^{2}-\omega^{2}\right)} .
$$

For most atoms the resonance absorption frequencies $\omega_{\alpha}$ are much larger than $\omega=\omega_{\text {laser }}=3 \cdot 10^{15} \mathrm{~s}^{-1}$ and hence $N-1=\varepsilon$ is a small positive number $\left(\varepsilon_{\text {air }}=2.7 \cdot 10^{-4}\right)$. Equation (3) holds also for a fully ionized plasma, where no bound electrons exist, when noticing $\omega_{\alpha}=0$ and introducing the plasma frequency via $\omega_{p e}=\sqrt{n_{e} e^{2} /\left(\varepsilon_{0} m_{e}\right)}$. In this case the index of refraction is less than unity and $N-1=\varepsilon \approx-0.5 \omega_{p e}^{2} / \omega^{2}$ becomes negative. However, inserting relevant values for the electron density $\left(n_{e}<10^{22} \mathrm{~m}^{-3}\right)$ the plasma frequency becomes as small as $\omega_{p e}<5.6 \cdot 10^{12} \mathrm{~s}-1$ and $\varepsilon$ is of order $10^{-6}$, i.e. much too small to contribute significantly. In other words, $N_{\text {ball }}$ can approach 1 with decreasing density but smaller values are not attainable. Let us assume that the limit $N=1$ is reached in the core region $r=0$. We then may consider (for $r \leq a$ ) the class of profiles

$$
N(r)=1+\varepsilon_{a i r}-\varepsilon_{a i r}\left(1-\frac{r^{2}}{a^{2}}\right)^{k}
$$

which, with rising parameter $\mathrm{k}$, attain $N(0)=1$ with increasing smoothness, as shown in Fig. 4 a. For $k=0$ a box-like shape with a sudden transition at the boundary $r=a$ is obtained. The corresponding profiles $\delta(\rho)$ as calculated from Eq. 1 are shown in Fig. 5 a. The case $k=0$ leads to the particularly simple result $\delta=2 \rho \varepsilon_{\text {air }} / \sqrt{a^{2}-\rho^{2}}$. Surprisingly, as to be seen in Fig. $5 \mathrm{~b}$, this fits best to our measured profiles when a ball radius of $a=8 \mathrm{~cm}$ is inserted. 

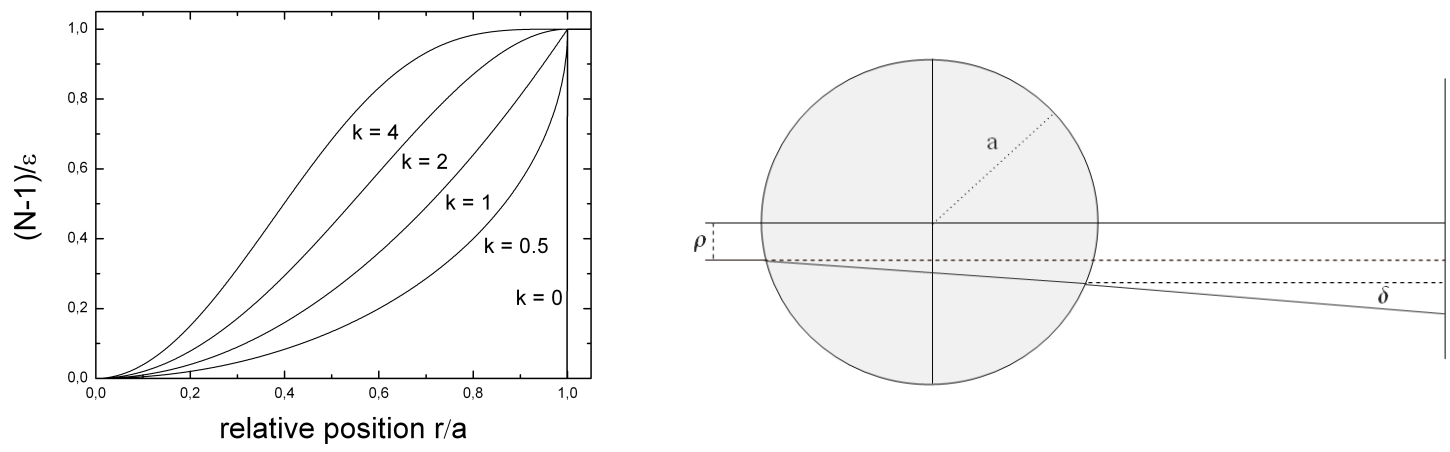

FIGURE 4. a) Index of refraction profiles considered b) Geometry of beam deflection
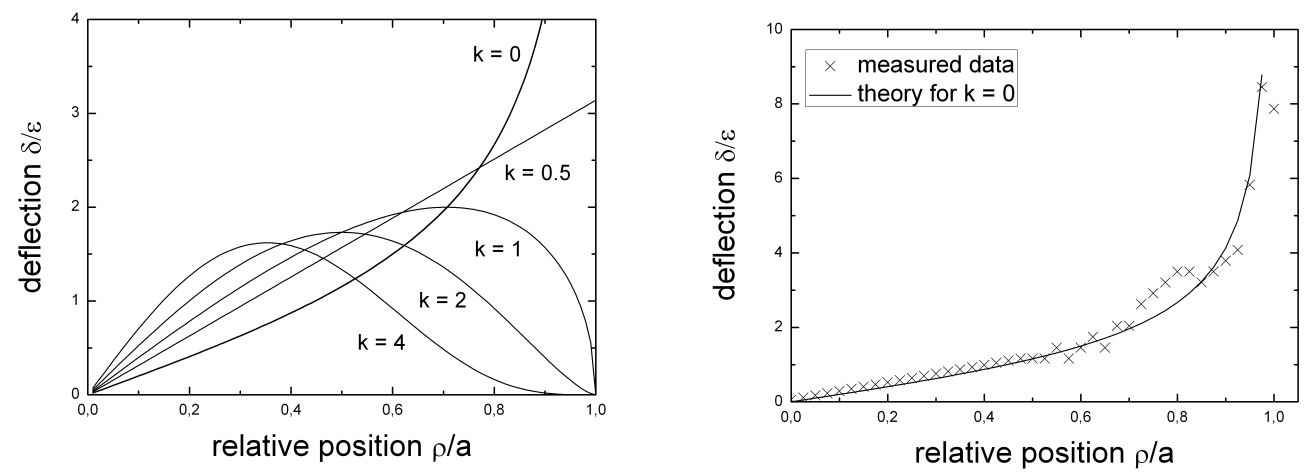

FIGURE 5. a) Angle of deflection profiles b) Comparison of measured data in vertical direction with the theory for $k=0$

\section{DISCUSSION}

The spectra are dominated by elements with small excitation energy, while spectral lines with energies of more than about $5 \mathrm{eV}$ are absent. This finding is in agreement with $T_{e} \leq 0.4 \mathrm{eV}$ as determined from Boltzmann plots. Positive charges could also be substantiated in the plasmoids from the occurrence of CaII and $\mathrm{MgII}$ lines. Ions of $\mathrm{Na}$ and $\mathrm{K}$ are likely to occur as well, although their spectral lines could not be detected. This is attributed to the rare gas electron configuration of these ions resulting in very high excitation levels (e.g. $32.8 \mathrm{eV}$ for NaII).

The index of refraction of the plasmoid is found to drop abruptly at the boundary towards values close to 1 . This indicates that the particle density within the ball like plasmoids decreases rapidly below the standard air value $n_{\text {air }}=6.7 \cdot 10^{25} \mathrm{~m}^{-3}$, such that the ratio $n_{\text {ball }} / n_{\text {air }}$ is smaller than approximately 8 . Invoking the ideal gas law $p=n k T$ we can then infer from pressure equilibrium $p_{\text {ball }}=p_{\text {air }}$ that the gas temperature in the plasmoid is rather high, $T>8 \cdot 273 \mathrm{~K}=2200 \mathrm{~K}$, a value consistent with our early measurements using thin thermocouples [4].

\section{REFERENCES}

1. A. A. Egorov, S. I. Stepanov, and G. D. Shabanov, Physics-Uspekhi 47, 99-101 (2004).

2. G. D. Shabanov, A. G. Krivshich, B. Y. Sokolovsky, and O. M. Zherebtsov, Proceedings 9th International Symposium on Ball Lightning, Eindhoven (2006).

3. B. Juettner, S. Noack, A. Versteegh, and G. Fussmann, "Long-living Plasmoids From a Water Discharge at Atmospheric Pressure," in Proceedings XXVIII International Conference on Phenomena in Ionized Gases, Prague 2007, 2007.

4. A. Versteegh, K. Behringer, U. Fantz, G. Fussmann, B. Juettner, and S. Noack, Plasma Sources Science and Technology (2007), to be published, see also ICPIC 2007.

5. W. Elmore, and M. Heald, Physics of Waves, Dover Publications, Mineola, N.Y., 1985. 